\title{
Magnetic Resonance Perfusion Imaging in the Diagnosis of High-Grade Glioma Progression and Treatment-Related Changes: A Systematic Review
}

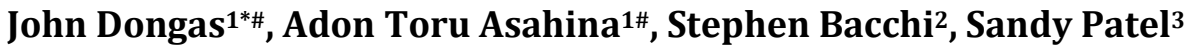 \\ ${ }^{1}$ University of Adelaide, Adelaide, Australia \\ ${ }^{2}$ Queen Elizabeth Hospital, Woodville, Australia \\ ${ }^{3}$ Department of Medical Imaging, Royal Adelaide Hospital, Adelaide, Australia \\ Email: *john.dongas@student.adelaide.edu.au
}

How to cite this paper: Dongas, J., Asahina, A.T., Bacchi, S. and Patel, S. (2018) Magnetic Resonance Perfusion Imaging in the Diagnosis of High-Grade Glioma Progression and Treatment-Related Changes: A Systematic Review. Open Journal of Modern Neurosurgery, 8, 282-305. https://doi.org/10.4236/ojmn.2018.83024

Received: May 11, 2018

Accepted: July 13, 2018

Published: July 16, 2018

Copyright () 2018 by authors and Scientific Research Publishing Inc. This work is licensed under the Creative Commons Attribution International License (CC BY 4.0).

http://creativecommons.org/licenses/by/4.0/

\begin{abstract}
In patients with high grade gliomas (HGGs), progression after treatment can be difficult to diagnose due to treatment-related effects, which overlap in appearance with tumour progression on conventional magnetic resonance imaging (MRI) sequences. Specialised imaging methods have been studied for this purpose, though most institutions currently use histopathology or clinicoradiological follow-up for diagnosis. This publication aims to review the evidence for perfusion MRI techniques. The databases of Pubmed, MEDLINE, EMBASE and Scopus were searched using combinations of the subject headings high grade glioma and MRI perfusion. 41 articles fulfilled the inclusion criteria. Dynamic Susceptibility Contrast (DSC) MRI was the most extensively studied, with several studies achieving high sensitivities and specificities. Other techniques exhibiting potential include Dynamic Contrast Enhanced (DCE) MRI, Arterial Spin Labelling (ASL). However, these techniques are not widely used or available for clinical practice. Composite measures combining results from multiple techniques tended to achieve higher accuracies. Some publications compared processing software used or looked at machine learning with relative success. An issue common to the literature is the lack of standardisation in the reference standard and acquisition/processing methods. Furthermore, many had small sample sizes, and further consideration needs to be given with regards to timing of imaging, and treatment regimens received in such studies.
\end{abstract}




\section{Keywords}

Magnetic Resonance Imaging, Perfusion, High Grade Glioma, Progression, Pseudo Progression

\section{Introduction}

High Grade Gliomas (HGGs), accounting for approximately $50 \%$ of all gliomas, are a group of highly malignant primary brain tumours (WHO Grades III and IV) known for their poor prognosis [1]. In patients with glioblastoma multiforme (GBM) particularly, overall median survival time is $14-18$ months, and progression occurs almost inevitably [2].

Currently, standard treatment for HGGs includes maximum safe tumour resection followed by concurrent chemotherapy and radiotherapy with adjuvant temozolomide (TMZ) as outlined by Stupp et al. [3]. However, with the addition of TMZ to the treatment regime, it is now believed that up to $30 \%$ of patients will show a new or enlarging area of enhancement on conventional magnetic resonance imaging (MRI), of which up to $64 \%$ may be attributed to treatment-related effects such as pseudo progression (PsP) or radiation necrosis ( $\mathrm{RN}$ ) [4] [5] [6].

These treatment-related effects are clinically important for several reasons. While true progression requires alteration of a patient's treatment regime, PsP may resolve spontaneously with continuation of the adjuvant TMZ, and is believed to carry a favourable prognosis [4] [5]. Furthermore, the validity of phase II clinical trials for salvage therapy in recurrent gliomas has been questioned due to the false inclusion of patients with PsP who appear to respond well to the new treatment, but are instead undergoing resolution of their treatment-induced changes [7] [8] [9].

Histopathology is the current gold standard for diagnosis of progressive disease. However, it suffers drawbacks including sampling error, surgical contraindications, and variable implementation of molecular classifications. Hence, a single non-invasive, imaging-based technique would be of clinical value. An earlier attempt to define treatment response known as the Macdonald Criteria did not recognise the clinical entity of treatment-related effects [10]. A later revision by the Response Assessment in Neuro-Oncology working group, known as the RANO criteria, attempts to account for PsP by limiting the diagnosis of true progression to times when an enhancing lesion occurs at least 12 weeks post-radiotherapy, or if the lesion is outside the confines of the radiation field [11]. However, this criterion is problematic since the enhancement almost always occurs within the radiation field, and it has been suggested that up to $30 \%$ of patients with PsP develop MRI changes more than 12 weeks after finishing radiotherapy [12]. 
Numerous trials have since looked at the potential of advanced imaging techniques for the distinction of these two clinical entities. Studies have examined the diagnostic ability of CT, conventional MRI, as well as advanced MR techniques including diffusion-weighted imaging (DWI) and MR spectroscopy, with various degrees of success. The evidence surrounding the use of perfusion MRI (pMRI) techniques to distinguish between true progression and treatment-related changes including PsP and RN will be reviewed here.

\section{Methods}

A systematic search of the databases of Pubmed, MEDLINE, EMBASE and Scopus was conducted with search terms: (high grade glioma OR Glioblastoma multiforme OR GBM) AND (MRI perfusion OR MR perfusion). The search was conducted on 14 April 2017. The articles were then filtered to isolate only those published in English (See Figure 1).

Eligibility determination and data extraction were performed independently by two authors (JD and AA). If a publication fulfilled the inclusion criteria, or if this could not be determined, the article was reviewed in full-text. In cases of disagreement, consensus was achieved through discussion, or review by an adjudicator (SB). The inclusion criteria were as follows: 1) Primary clinical publication (not a review/editorial-assessed humans rather than cell or animal model) (excluding individual case reports and unique conference abstracts), 2) Involved patients with high-grade glioma (grade III and IV) that had been treated (not surgery alone-e.g. including, but not limited to, chemotherapy or radiotherapy), who received MR perfusion imaging, 3) Reported on the effectiveness (including, but not limited to, sensitivity/specificity) of any parameter of MRI perfusion (either alone or as a part of a composite measure) to distinguish tumour progression from differentials (such as radionecrosis or PsP), and 4) Available in full text.

Data extraction was performed in duplicate using a standardized table by two authors (JD and AA). Risk of bias was assessed using a combination of Cochrane and QUADAS assessment tools. Glioma progression was considered as positive, and relevant differentials as negative.

\section{Results}

A total of 1135 potential articles were identified in the initial search. 1089 of these publications were written in English. After examining titles and abstracts, 328 publications were reviewed in full-text. A further 287 articles were excluded at this stage based on the inclusion/exclusion criteria, and the remaining 41 articles are included in this review (see Figure 1).

The results of the critical analysis for bias and quality using the Cochrane and QUADAS assessment tools is summarised in Table 1. The overall quality for each of the publications was satisfactory for final inclusion. Most issues were associated with inherent limitations of retrospective analyses (such as lack of 


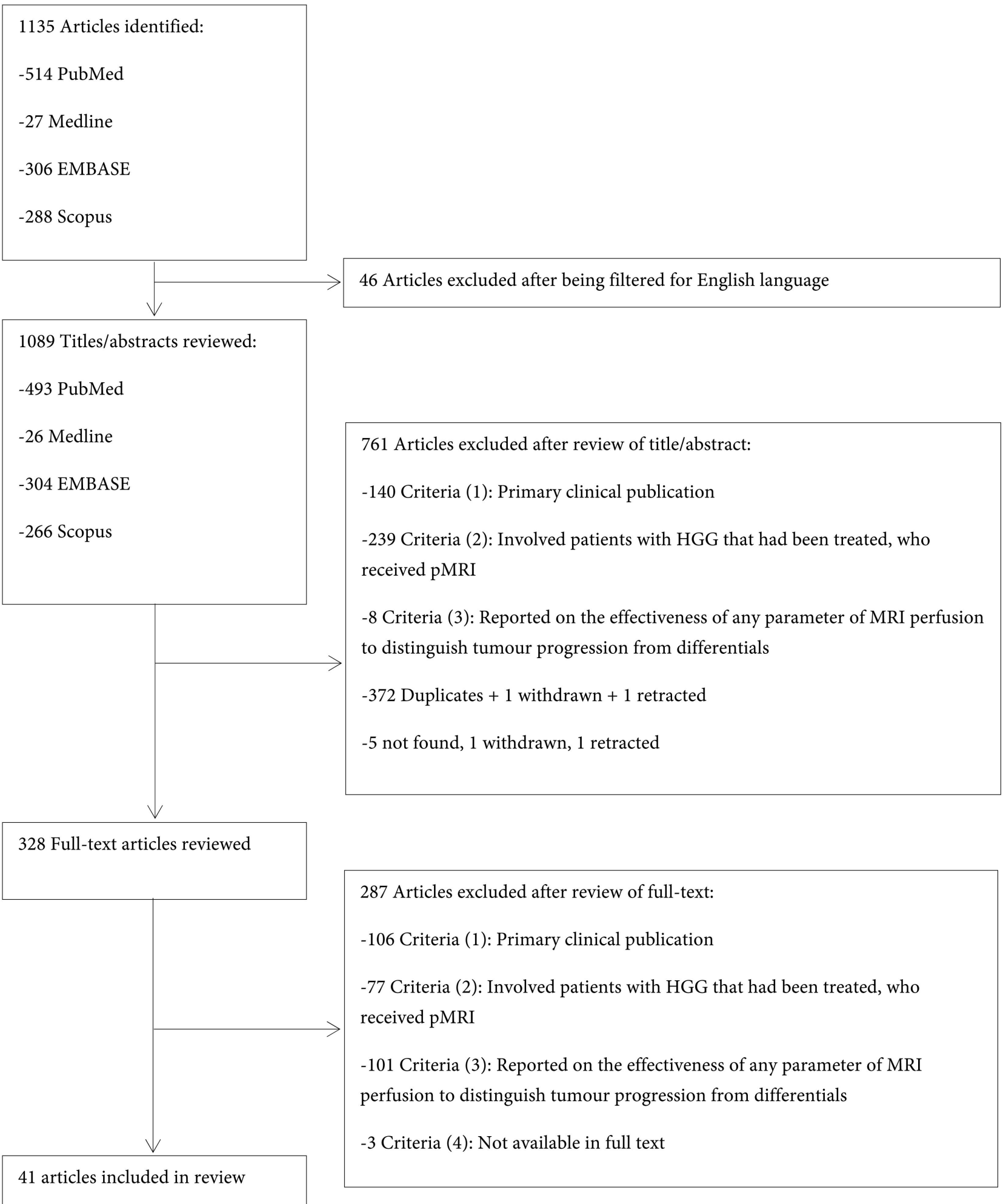

Figure 1. Flowchart detailing results from the search strategy and application of inclusion and exclusion criteria.

standardisation of treatment and imaging follow-up received) or inconsistency in the reference standard (e.g. histology vs clinicoradiological follow-up). Studies adequately defined the exact MRI technique and parameters used, with one main 
Table 1. Summary of quality analysis and bias results according to QUADAS and Cochrane methods.

\begin{tabular}{|c|c|c|c|c|c|c|c|}
\hline \multirow[b]{2}{*}{ Study } & \multicolumn{4}{|c|}{ Risk of Bias } & \multicolumn{3}{|c|}{ Applicability Concerns } \\
\hline & $\begin{array}{l}\text { Patient } \\
\text { Selection }\end{array}$ & $\begin{array}{c}\text { Index } \\
\text { Test }\end{array}$ & $\begin{array}{c}\text { Reference } \\
\text { Standard }\end{array}$ & $\begin{array}{c}\text { Flow and } \\
\text { Timing }\end{array}$ & $\begin{array}{c}\text { Patient } \\
\text { Selection }\end{array}$ & $\begin{array}{c}\text { Index } \\
\text { Test }\end{array}$ & $\begin{array}{l}\text { Reference } \\
\text { Standard }\end{array}$ \\
\hline Barajas et al. 2009 & & & 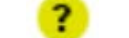 & & & & \\
\hline Blasel et al. 2016 & & & & & & & $?$ \\
\hline Choi et al. 2013 & & & & & & & \\
\hline Dandois et al. 201 & & ? & & & & & \\
\hline Kim TH et al. 201? & & ? & & & & & \\
\hline Ozsunar et al. 201 & & & : & & & & \\
\hline Prager et al. 2015 & & & & & & & \\
\hline Shin et al. 2014 & & ? & & & & & " \\
\hline Wang et al. 2016 & $?$ & ? & & & & & ? \\
\hline Seeger et al. 2013 & & & ? & & & & \\
\hline Heo et al. 2015 & & & & & & & \\
\hline Kim et al. 2014 & & & & & & & \\
\hline Park et al. 2016 & & & & & & & \\
\hline Sacconi et al. 2016 & & & & & & & \\
\hline Hamilton et al. 201 & & & & & & & \\
\hline Kelm et al. 2015 & & $?$ & & & & & \\
\hline Song et al. 2013 & & & & & & & \\
\hline Yoo et al. 2016 & & & & & & & \\
\hline
\end{tabular}

† Low Risk, †High Risk, ? Unclear Risk.

exception, which referred only to "perfusion MRI" [13]. 26 of the included studies examined a parameter of Dynamic Susceptibility Contrast (DSC) MRI [14]-[39]. 12 studies examined Dynamic Contrast Enhanced (DCE) MRI [34] [35] [40]-[49]. Arterial Spin Labelling (ASL), a non-contrast perfusion technique, was studied in 3 studies [19] [31] [34]. 10 articles looked at composite/multiparametric measures using either multiple perfusion parameters or 
combinations of advanced MR techniques including MR spectroscopy or DWI [19] [21] [27] [32] [33] [34] [38] [42] [43] [50]. A further 3 articles looked at aspects of information technology; including different software processing methods, and also machine learning [25] [51] [52]. At least 8 made comments on inter-observer reliability [17] [20] [37] [41] [42] [43] [48] [52].

\subsection{Dynamic Susceptibility Contrast (DSC) MRI}

DSC-MRI involves the administration of a gadolinium-based contrast agent (GBCA) followed by the rapid acquisition of gradient or spin echo images, dynamically capturing the transient decrease in signal intensity caused by the local magnetic field distortion of the GBCA within and around blood vessels [53]. By processing the attained signal intensity (SI)-time curve, various perfusion parameters including cerebral blood volume (CBV), cerebral blood flow (CBF) and mean transit time (MTT) can be obtained. These values are generally normalised to the contralateral, normal-appearing white matter (NAWM). Any contrast leaving the vascular compartment will prevent signal from returning to baseline.

Of the studies examining DSC-MRI, 23 looked at CBV values [14]-[39], by far the most common perfusion parameter studied in the literature. Three studies used a histogram-based analysis [15] [26] [37]. Two publications looked at parameters from the SI-time curve including relative peak height $(\mathrm{rPH})$ and percentage signal recovery (rPSR) [16] [39]. There were 3 studies that performed qualitative analysis using maps obtained from DSC-MRI [19] [31] [36].

Although several methods exist, the relative CBV (rCBV) for a given region of interest (ROI) is generally calculated by evaluating the negative enhancement integral to estimate the area under the SI-curve during the first pass of contrast agent (see Figure 2 for examples of these measurements) [53]. The rCBV is expected to be higher in progressive gliomas due to the extensive neovascularisation, increasing the intravascular compartment volume per voxel. In their article, Alexiou et al. [14] reported a sensitivity and specificity of $100 \%$ for rCBV using a cut-off value of $2.2 \mathrm{~mL} / 100 \mathrm{~g}$ in a cohort of 30 patients. These results have also been obtained in two other studies, although with much smaller sample sizes of 9 and 10 respectively [24] [28]. Most other studies provided $P$ values and reached statistical significance for the use of $\mathrm{rCBV}$ to distinguish true progression and treatment-related effects with sensitivities ranging from 63\% [38] to $100 \%$ [14] [18] [24] [28] [33] and specificities from 67.9\% [19] to 100\% [14] [18] [20] [23] [24] [28] [39]. Among these studies however, optimum cut-off values ranged extensively from 0.71 according to $\mathrm{Hu}$ et al. [24], to as high as 5.01 in Sacconi et al. [33]. Unfortunately, the issue of wide variability in cut-off values is known to the literature because of a lack of standardisation in acquiring and processing rCBV values, including different scanner technologies, varying algorithms for processing, and at times, a lack of quantification. Other reasons for this variability included the use of maximum (instead of mean) rCBV values [17] [19] [27] [33] [38], or the choosing of specific ROIs with high CBV within a lesion, rather than looking at the entire enhancing area. One study by Prager et al. 


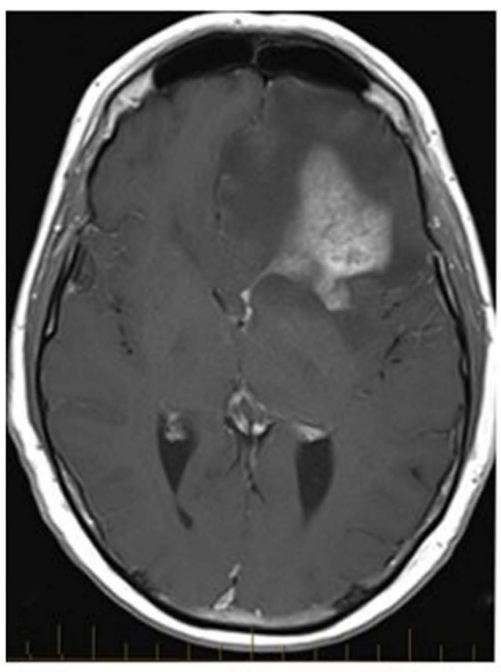

(a)

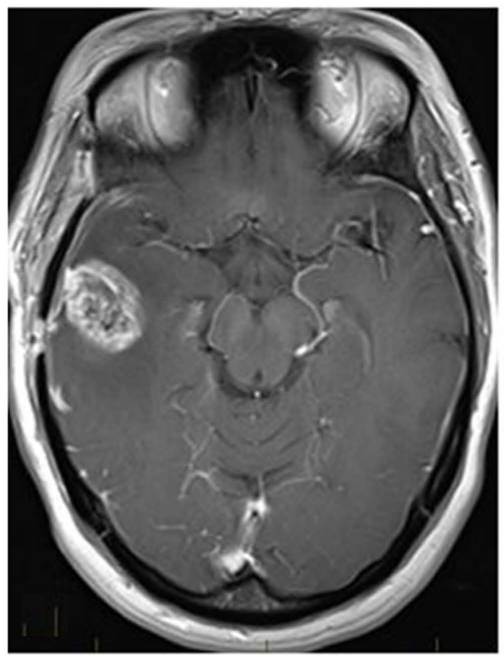

(c)

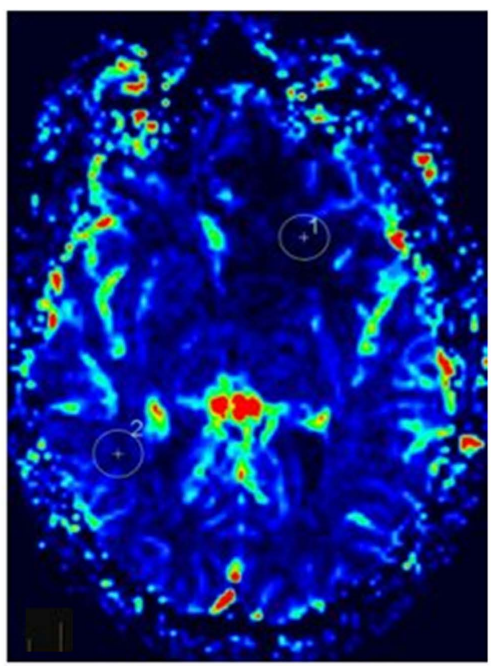

(b)

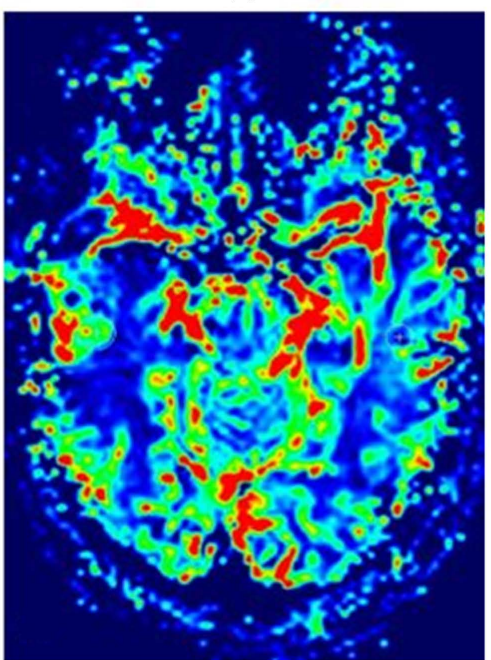

(d)

Figure 2. Examples of rCBV calculation using DSC imaging in 2 patients with GBM. Patient 1 -imaging done 2 weeks post-completion of initial therapy (surgery + CCRT). (a) $\mathrm{T} 1$ post-contrast image shows an enhancing left anterior frontal mass; (b) DSC perfusion map at same level with left frontal mean value CBV 69.5, and right posterior temporal mean value $\mathrm{CBV}$ 97.5. $\mathrm{rCBV}=0.71$ (69.5/97.5), consistent with pseudo progression. $\mathrm{PET}$ scan supportive. mass subsequently reduced in size and enhancement. Patient 2 -imaging done 3 months post-completion of initial therapy (surgery + CCRT); (c) T1 post-contrast image shows enhancing mass in right temporal lobe; (d) DSC perfusion map with right temporal mean value CBV 1041.3 and left temporal mean value CBV 510.9. $\mathrm{rCBV}=2.0$ (1041.3/510.9), consistent with recurrent tumour. Diagnosis proven with surgery.

[32] showed how a higher optimum cut-off (1.74) can be obtained when calculating the rCBV of chosen ROIs instead of the whole lesion (1.27). Despite the existing evidence favouring the use of $\mathrm{rCBV}, 5$ studies failed to reach statistical significance in at least one of the parameters they examined [18] [28] [29] [33] [37]. A summary of the most robust studies looking at CBV, including those with a sample size greater than 20 , low risk of bias, attempt to incorporate histological verification, and clear acquisition parameters, are summarised in Table 2. 


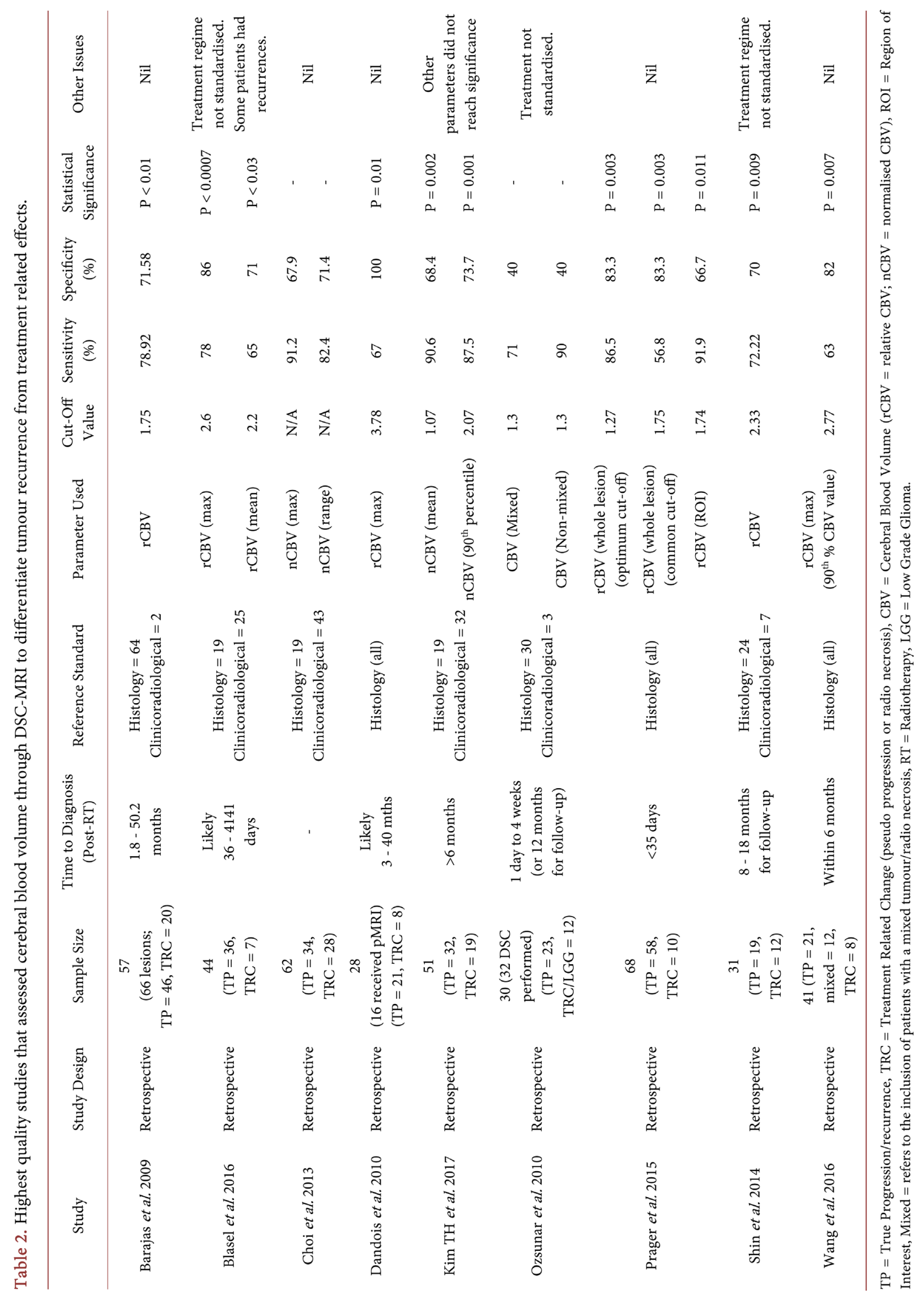


Kim et al. [26] performed a study looking at parameters derived from a normalised rCBV histogram such as histogram width (HW), maximum frequency (PHP) and maximum value (MV). These parameters gave 92.3\%, 90.2\% and $91.5 \%$ for sensitivity, and $70.5 \%, 91.1 \%$ and $72.5 \%$ for specificity respectively for differentiating tumour progression from mixed and treatment-effect groups in a cohort of 39 patients. In a similar study of 20 patients, Song et al. [37] looked at the $95^{\text {th }}, 90^{\text {th }}$ and $75^{\text {th }}$ percentile points of the histogram, as well as the PHP. They were not able to replicate success and only one observer obtained statistical significance $(\mathrm{p}=0.015)$ for the $95^{\text {th }}$ percentile cut-off. Baek et al. [15] looked at several parameters including maximum, mode, range, as well as percent change in skewness and kurtosis from first to second follow up MR images. All parameters reached statistical significance with a negative change in skew the best predictor, attaining a sensitivity and specificity of $85.7 \%$ and $89.2 \%$ respectively.

Relative $\mathrm{PH}(\mathrm{rPH})$ and relative PSR (rPSR) are two parameters determined from the $\mathrm{T} 2^{\star}$-weighted SI curve. $\mathrm{PH}$ is the difference between the pre-contrast and minimum signal intensity during first pass of the contrast agent, whilst PSR refers to the amount of signal intensity recovered based on the difference between pre- and post-contrast signal intensity, and expressed as a percentage. Barajas et al. [16] obtained a sensitivity of $89.32 \%$ and specificity of $81.38 \%$ using an $\mathrm{rPH}$ cut-off of 1.38 in 79 patients. rPSR did not perform as well. In another article by Young et al. [39], rPH > 1.7 was $100 \%$ sensitive and specific for progressive disease, while again, rPSR was inferior yet still highly specific with $63 \%$ sensitivity and $100 \%$ specificity, albeit using a much smaller cohort of 20 patients.

Finally, qualitative analysis using $\mathrm{rCBV}$ maps was conducted as part of 3 studies. In Choi et al. [19], colour gradients within the ROI were compared to other areas such as white matter, grey matter or blood vessels, to determine whether there was definitely increased perfusion in the enhancing area. They obtained $82.4 \%$ sensitivity and $67.9 \%$ specificity though they failed to reach statistical significance $(\mathrm{p}=0.133)$. Ozsunar et al. obtained similar sensitivity and specificity of $86 \%$ and $70 \%$ respectively, though they did manage to reach significance in their results $(\mathrm{p}<0.01)$ [31]. A more recent publication by Snelling et al. [36] in turn found a sensitivity of $60.8 \%$ and specificity of $87.8 \%$ using a purely qualitative method.

\subsection{Dynamic Contrast Enhanced (DCE) MRI}

Like DSC imaging, DCE-MRI also involves administration of a GBCA. It depends, however, on the T1-shortening effects of the contrast agent as it accumulates within the tissue extracellular space at a rate defined by properties of the blood brain barrier including perfusion, permeability and surface area [53].

After defining a vascular input function (VIF), complicated pharmacokinetic modelling can be used to yield fully quantitative parameters such as extravascular, extracellular $\left(\mathrm{V}_{\mathrm{e}}\right)$ and plasma $\left(\mathrm{V}_{\mathrm{p}}\right)$ volumes, as well as a transfer constant $\left(K^{\text {trans }}\right)$, which is believed to reflect microvascular permeability, blood flow, and 
surface area [54]. Progressive tumours are expected to have higher $V_{p}$ and $K^{\text {trans }}$ because of extensive angiogenesis and leaky vasculature. This approach was taken in 6 of the included studies [34] [35] [41] [44] [47] [48]. A study by Hamilton et al. [41] gave an overall accuracy of $79 \%$ for four parameters. Mean and $\max \mathrm{K}^{\text {trans }}$ as well as mean $\mathrm{V}_{\mathrm{e}}$ gave $80 \%$ sensitivity and $78 \%$ specificity while mean $\mathrm{V}_{\mathrm{p}}$ gave $71 \%$ sensitivity and $89 \%$ specificity using cut-offs of $0.05,0.2,2$ and 12 for each of these parameters respectively. Their study also revealed that a VIF derived from the superior sagittal sinus (SSS) performs better than that taken from the middle cerebral artery [41]. Two other studies achieved similar results using $\mathrm{K}^{\text {trans }}$ [34] [48] though only Yoo et al. also reached significance using the mean $\mathrm{V}_{\mathrm{e}}$ with a cut-off of 0.873 [48]. Shin et al. [35] normalised their values to the contralateral NAWM and obtained a sensitivity of $61.1 \%$ and specificity of $80 \%$ using a cut-off of 2.1 for the mean $\mathrm{K}^{\text {trans }}$. Significance was also obtained in a study by Thomas et al. [47], which obtained significance using the normalised mean and $90^{\text {th }}$ percentile points for $\mathrm{V}_{\mathrm{p}}$. The latter performed best with a $92 \%$ sensitivity and $85 \%$ specificity. There was one study that obtained CBV values using a Patlak plot [55]. In this article, Larsen et al. [44] achieved $100 \%$ sensitivity and specificity for detecting tumour progression. However, it should be noted that this was using a small cohort of 14 patients, of which only 2 were diagnosed with treatment-related effects.

There were 8 studies which used semi-quantitative descriptive indices from the raw signal intensity data as plotted on the SI-time curve; parameters which are less physiologically specific, but still reflect the CBF, CBV and microvascular permeability [35] [40] [41] [42] [43] [45] [46] [49]. Chung et al. [40] have suggested that the initial area under the curve (IAUC) may be higher with the increased tumour vascularity and surface area, while treatment-related effects might show a higher final AUC (FAUC) due to greater retention of contrast in the extracellular, extravascular space associated with cell loss and tissue damage. In their study, they obtained statistical significance for several mean and $90^{\text {th }}$ percentile point parameters, with a best of $93.8 \%$ sensitivity and $88 \%$ specificity for the mean AUC ratio of IAUC (0 - $30 \mathrm{~s})$ to FAUC (320 - $350 \mathrm{~s})$ using a cut-off of 0.23 [40]. These results were replicated in a study by Suh et al. [46] which looked at the AUC ratio and found $90.1 \%$ sensitivity and $82.9 \%$ specificity with a cut-off of 0.31 . Several studies have demonstrated the validity of the $90^{\text {th }}$ percentile point of IAUC [42] [49]. The $90^{\text {th }}$ percentile histogram is believed to be more statistically reliable, yet almost equivalent to the maximum value [40]. Interestingly, Heo et al. [42] obtained their results in a cohort of patients for whom DSC-MR images were uninterpretable. Others have validated the use of mean IAUC [35] [41] [45]. Hamilton et al. [41] used what they termed "delayed short AUC" (the AUC between end of the initial wash-in phase the start of the early washout phase) divided by the VIF to obtain $93 \%$ sensitivity and $78 \%$ specificity with a cut-off ratio of $20 \%$. Finally, Narang et al. [45] examined two different semi-quantitative parameters, the slope of the delayed equilibrium phase (SDEP) and maximum slope of initial vascular phase (MSIVP). The latter obtained a 
sensitivity and specificity of $100 \%$ with a cut-off value of 9.5 in their cohort of 29 patients.

\subsection{Arterial Spin Line Imaging (ASL)}

Three publications reported on arterial spin labelling (ASL) for differentiating HGG progression from differentials [19] [31] [34]. ASL measures perfusion using the patient's own water molecules as tracers, and hence does not require the administration of gadolinium contrast agents. A 3 point qualitative grading assessment of ASL was found to have equal [19], or greater [31] diagnostic accuracy compared to quantitative assessment. These studies are summarised in Table 3 .

\subsection{Composite/Multiparametric Measures}

Of the 10 studies that used composite/multiparametric MRI, 6 used solely DSC [21] [27] [32] [33] [38] [50], 1 used solely DCE [42], 1 used ASL + DSC [19], 1 used DSC + DCE [43], and 1 used ASL + DSC + DCE [34] adjunctive to other non-perfusion based techniques. In general, the composite measures exhibited greater diagnostic accuracy compared to single parameters, except for 3 studies which found no statistically significant difference for certain measures [19] [32] [33]. Further, studies utilising three or more parameters in a single measure had greater diagnostic accuracy when more imaging modalities were utilised compositely (see Table 4) [21] [34] [50].

\subsection{Software Processing/Machine Learning}

Three studies reported on aspects related to computer software in improving diagnostic accuracy. Two studies focused on differing software processing [51] [52], and one looked into the use of machine learning via an automatic support vector machine (SVM) in place of a human index test assessor [25].

$\mathrm{Hu}$ et al. [51] and Kelm et al. [52] utilised different software packages including IB Neuro (IBN), Nordic ICE (NICE) and FuncTool to synthesise CBV images from DSC-MRI, and subsequently assess their ability to differentiate tumour progression from treatment-related change and pseudo progression. IBN and NICE employ the use of the Boxerman mathematical algorithm, whereas FuncTool utilises pre-bolus and post-bolus linear interpolation in calculation [52]. Hu et al. [51] obtained an optimal accuracy of $85 \%$ with a cut-off of $50 \%$ fractional tumour burden through IBN correction of rCBV, compared to NICE correction with gamma variate fitting (gvf) (67\%) and without gvf (70\%). This was in a cohort of 52 patients, with significant variation in initial tumour histology, and contrast materials. In contrast, Kelm et al. [52] assessed accuracy and variation in results between software with a number of different threshold values, 1.5T/3T imaging, and classification criteria (e.g. survival, treatment change and combined). It was found that there was always one software package as outlier for each of the three-metrics compared to the other two, a different package being outlier for each. This suggests considerable variability between software. 


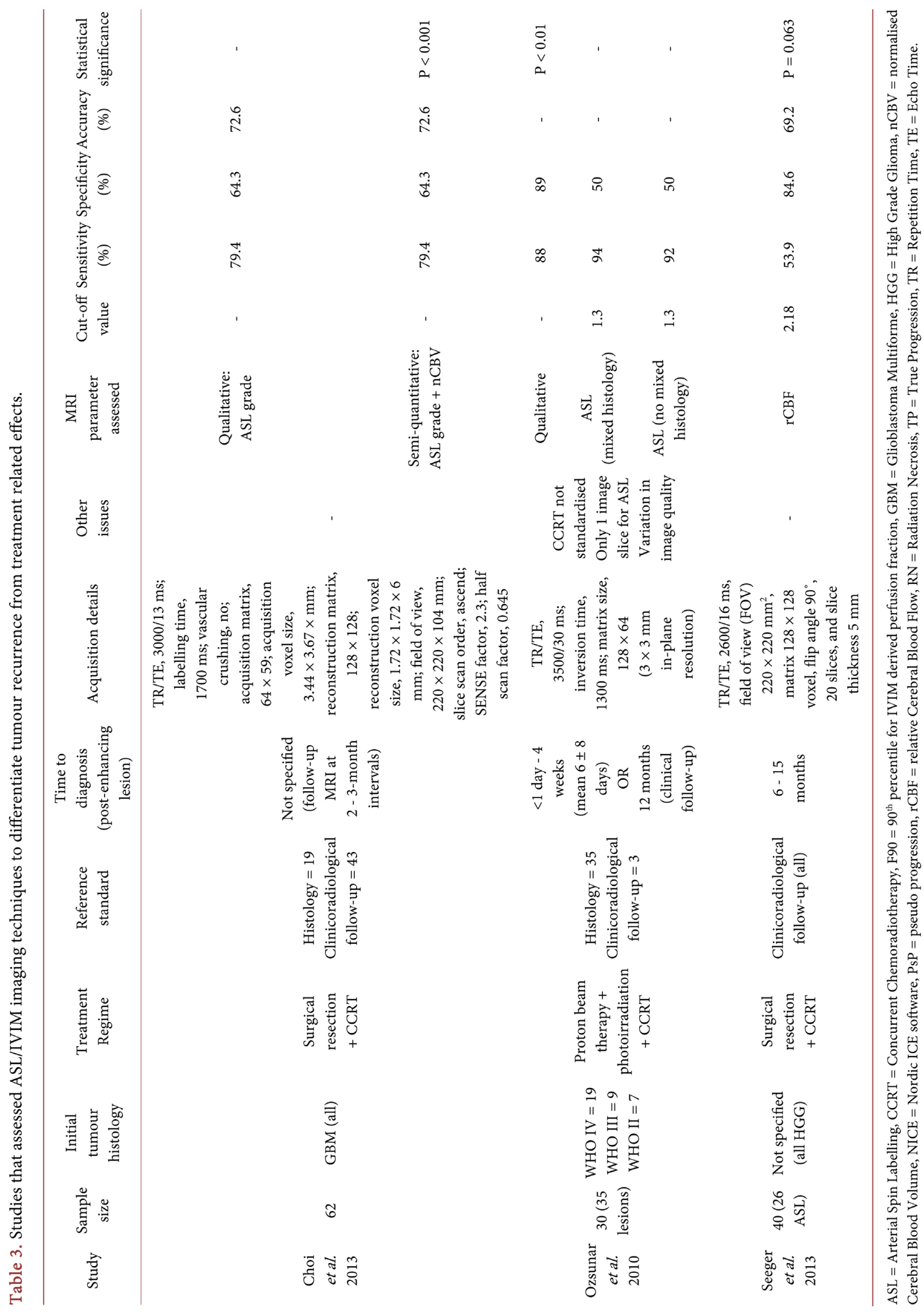




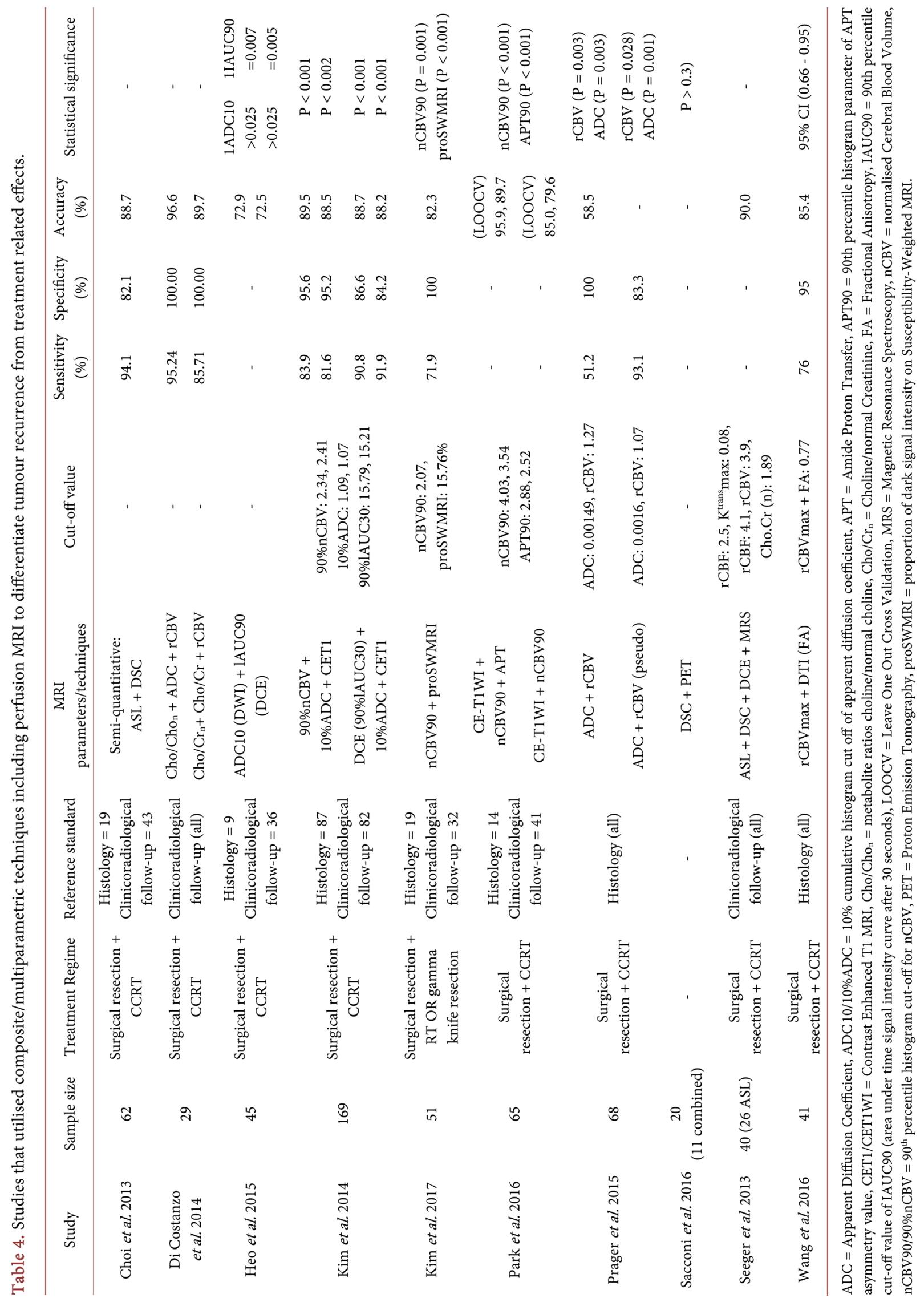


However, use of survival and treatment change as the reference standard inherently gives wide variability compared to histopathology. As such, the authors could not recommend an optimal choice. Suggested/published cut-offs and processing methods specific to each of the software packages should be adhered to.

An automatic SVM to replace the index test assessor was utilised by $\mathrm{Hu}$ et al. [25] in 31 patients with GBM. An optimal nCBV and nCBF cut-off of 1.14 and 0.98 respectively garnered sensitivities of $89.1 \%$ and $84.4 \%$, and specificities of $87.7 \%$ and $91.0 \%$. This method has the clinical advantages of being fully automatic (save for choosing a ROI in contralateral tissue), with decreased operator dependence/time input, and respectable diagnostic accuracy. Larger trials are required to confirm these results.

\subsection{Interobserver Variability}

Eight publications had two or more separate outcome assessors to assess inter-observer variability. The majority of studies assessing DSC-MRI exhibited satisfactory inter-reader correlation [20] [37] [43] [52], with one study showing high correlation $(r=0.932 \mathrm{p}<0.001)$ for manual drawn ROIs [17]. DCE parameters similarly showed satisfactory inter-reader concordance in all 3 studies in which it was assessed [41] [42] [43] [48]. Some studies showed how reliability could differ among specific parameters within DSC and DCE [41] [43]. A summary of the inter-observer reliability can be found in Table 5.

\section{Discussion}

The lack of an appropriate diagnostic criteria that properly accommodates the clinical entity of post-radiotherapy effects indicates that a non-invasive, imaging based, diagnostic tool would be useful in their distinction from progressive glioma. In this article, the evidence for various parameters of perfusion-based MRI techniques including DSC, DCE, and ASL MRI, as well as composite measures with other advanced MRI techniques have been reviewed. In addition, the review has encompassed the use of machine learning, various software packages, as well as the inter-observer variability of these techniques.

One of the difficulties with perfusion-based MRI techniques is that of identifying the dominant pathology in the setting of heterogeneous changes (for example, combinations of HGG, LGG, necrosis, and inflammation in an individual patient). Another issue is the lack of standardisation of acquisition parameters and processing techniques. Problems related to acquisition include field strength, gradient echo versus spin echo, flip angle, use of contrast agent, and issues inherent to the specific perfusion technique. Firstly, the use of higher magnet strengths i.e. $3 \mathrm{~T}$ vs $1.5 \mathrm{~T}$ gives several advantages, including a better spatio-temporal resolution, and a higher signal-noise ratio [56] [57]. 3T over 1.5T has been validated in one of the publications included in this study [52]. Unfortunately, 3T systems are not available at all institutions. Although gradient-echo 


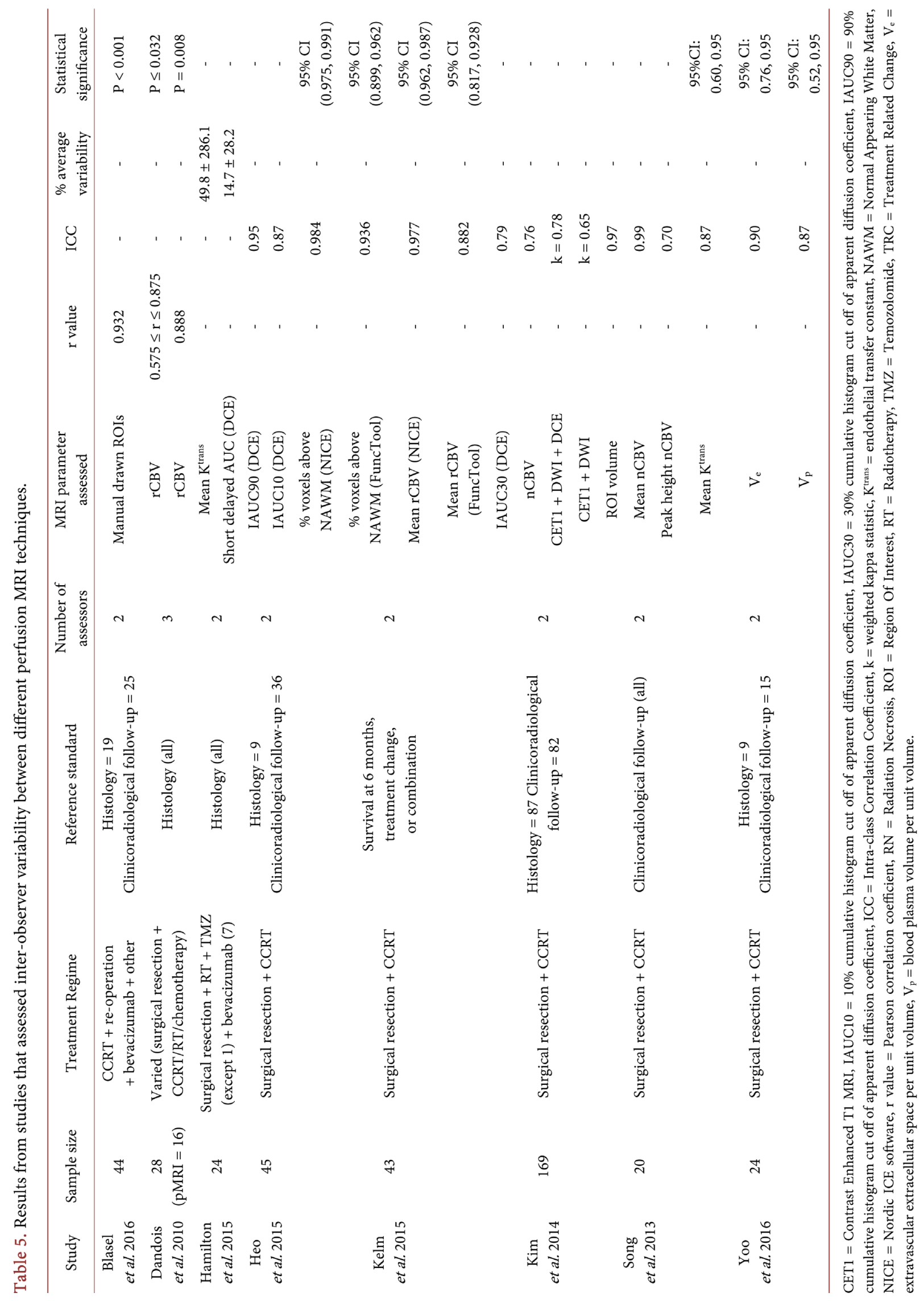


acquisition of DSC images is the most preferred, and widely used technique, it has been suggested that spin-echo acquisition may offer a higher signal-noise ratio, and reduced susceptibility artefact [51]. Spin-echo requires higher doses of contrast, and there is insufficient evidence to suggest that spin-echo is superior for diagnosing tumour progression in GBM patients [17] [51].

Furthermore, choice of contrast agent can also affect the results. For example, gadobenate has exhibited twice the $\mathrm{T} 1$ relaxivity effects compared to gadodiamide, suggesting that the former would exhibit a larger T1 leakage effect [58]. This pertains particularly to DSC-MRI where a leaky BBB means that T1 leakage and $\mathrm{T}^{*}$ residual effects must be corrected in order to achieve maximum accuracy. Administration of a preload dose of contrast before the main bolus can saturate the interstitium, partially correcting for these effects, but the extent to which it does so remains undefined [17]. Mathematical correction techniques can also be employed. These include the use of a smaller flip angle (FA), mathematical techniques such as gvf, baseline subtraction, or other software-based algorithms. Unfortunately, low FA reduces the signal-noise ratio and can potentially exacerbate $\mathrm{T} 2{ }^{*}$-weighted effects [59]. These techniques were variably implemented in the reviewed studies, with the majority using pre-load contrast dosing [17] [18] [20] [21] [23] [24] [31] [35] [38], or a small 35 FA [15] [16] [19] [26]. Post-imaging techniques were also utilised in most studies including gvf [15] [19] [26] [27] [32] [35] [37] [38], baseline subtraction [23], or software based algorithms [14] [17] [18] [24] [27] [30] [31] [35] [37]. 7 studies did not specify, or were unclear about what technique was used [22] [25] [29] [33] [34] [36] [39]. A comparison between these techniques as it pertains to the diagnosis of tumour progression, or at least some method of standardisation, is required. The method for normalisation must also be standardised, since grey and white matter have different microvasculature features. Gahramanov et al. [60] have proposed the use of an iron-based agent, ferumoxytol, since it exhibits less extravasation, and hence leakage correction is not an issue. Furthermore, ferumoxytol is not renally cleared and so may have other advantages in patients with renal failure or contrast allergies. Additionally, DSC-MRI has other inherent limitations including high susceptibility to artefact, especially in areas around the skull base, from blood products from surgery, or from metallic hardware. Another primary inconsistency in the included studies is the choice of the ROI. Whilst some used a "hotspot" method, others used the entire enhancing lesion, potentially reducing the $\mathrm{rCBV}$ in these areas. At present, there is no widely available technique to accurately calculate $\mathrm{CBV}$ in $\mathrm{ml} / 100 \mathrm{~g}$ brain on MRI, as is currently achievable on CT. Most centres therefore employ rCBV measurements. Standardisation is required in the first instance, however ultimately, the preferred option would be a validated, and widely accepted quantitative technique.

DCE-MRI has higher spatial resolution and is more resistant to degradation from susceptibility artefact from bone, air and blood interfaces. However, its processing is more complicated. Model-based pharmacokinetic processing, such 
as that outlined by Tofts et al. [54] presents several difficulties despite supposedly giving greater and more specific physiological insight [61]. These difficulties include parameter coupling, measuring of the vascular input function, water exchange, and model fit instability [19]. Among the included studies, Hamilton et al. [41] suggested that use of the SSS for the VIF maximised accuracy for tumour progression diagnosis; however, values can still vary largely if care is not taken. Inconsistencies still exist in using the semi-quantitative parameters. For example, the IAUC was determined using variable time points such as $0-30$ s or as long as 120 s.

Although ASL has its own advantages including reduced susceptibility artefacts, low cost and no need for GBCA, it is not yet widely accepted in its own right and suffers from reduced signal-noise [19] [31] [62]. As for the use of composite measures, acquisition times and costs need to be considered, as well time taken for data processing, and difficulty in interpretation, even amongst expert readers.

Another issue to be considered is the difference between PsP and radiation necrosis. While PsP usually occurs early (within the first 3 months, though may be longer), late radiation necrosis in particular may occur months or years following treatment. Some studies have suggested that the timing of MRI following treatment should be taken into account when interpreting images since the two might require different cut-off values [48]. Shin et al. [35] suggested that PsP is more difficult to distinguish from progression compared to radiation necrosis. Likewise, methylation of the $\mathrm{O}^{6}$-methylguanine-DNA methyltransferase (MGMT) promoter may need to be considered since it has been suggested in a recent study that the MGMT protein may modulate angiogenesis, and hence, alter the rCBV of a lesion [49] [63].

It has already been mentioned that there is a lack of standardisation in the reference standard for assessment of treatment response. This inconsistency is reflected as a weakness in many of the studies that used both histology and clinicoradiological follow-up for diagnosis. Histology is the current gold standard; however, surgical access is not always feasible and it may carry morbidity rates from $1 \%-4.7 \%$, as well as mortality rates from $0 \%-2.3 \%$ [14] [16] [64] [65]. Even when possible, biopsy may give false negatives due to a $10 \%$ tissue sampling error [66]. There is considerable variability in co-registering regions of histology with ROIs on MRI. Ideally, a simple grid pattern would be undertaken through the resected lesion and co-registered to an identical grid on imaging. However, this presents challenges given inherent variation in surgical techniques, lesion morphology, lesion location and tissue distortion in vitro. Significantly, in approximately one third of patients, histology shows a mixture of tumour progression and radiation effects [67]. Some of the included authors have acknowledged this issue, and attempted to correlate perfusion MRI with a so-called "fractional tumour burden". How this might impact clinical decision-making, such as when to alter treatment regimens, remains to be seen. As 
for follow-up, studies are limited by the length of the follow-up period. Indeed, patients may develop treatment-related changes, and also progression concurrently. This may confound results depending on follow-up time and diagnostic criteria used in particular publications.

Aside from the limitations that have already been discussed, many of the studies had a small sample size, particularly in the treatment effect group. This may call into question the validity of the specificities obtained in these studies. Furthermore, some studies had other primary goals such as determining overall survival, with differentiating progression and $\mathrm{PsP} /$ radio necrosis as a subsection. This may give rise to selective reporting bias, without full exploration of their results. Other potential issues include the heterogeneity of tumour types and grades included, with some studies incorporating small numbers of low-grade tumours in their cohorts. The use of additional treatments such as steroids or bevacizumab in some studies may also affect perfusion parameters.

Future studies in this area should endeavour to use larger sample sizes, and also seek to compare acquisition and processing techniques so that the best methods can be obtained for standardising purposes. Vendor cooperation with regards to standardisation of scanner technology and software would facilitate this aim. High quality studies should use a robust and consistent criterion for the reference standard; either histology or long-term follow-up. Details of the patient cohort and timing of MRI in relation to the chemoradiotherapy treatment should be made clear. Physicians seeking to apply perfusion MRI into their clinical practice should endeavour to adhere to the same pMRI technique, acquisition parameters, and processing techniques as the authors of the relevant publication when applying cut-off values cited in an article.

It should be acknowledged that this review has several limitations, such as the exclusion of studies not published in English. Furthermore, although inclusion of conference abstracts is desirable to minimise the potential for publication bias, these were excluded from the review since it was felt that details of the image acquisition and processing are important considerations, and that insufficient detail would be available from conference abstracts.

\section{Conclusion}

Perfusion MRI is one of the imaging techniques with the potential to differentiate tumour progression from treatment-related effects including pseudo progression and radiation necrosis. DSC-MRI is the most well-studied. Other potential candidates include DCE-MRI and ASL techniques. Although each has shown promise, no single technique has been shown to be reliable as of yet. Future studies should aim to use larger patient cohorts with standardisation of treatment regimes, as well as acquisition and processing methods. Refinement of algorithms to combine multiple parameters is likely to achieve higher sensitivity and specificity than single parameter assessment owing to the heterogeneity of the pathology and imaging challenges. 


\section{Conflict of Interest}

The authors declare that they have no conflict of interest.

\section{Funding}

This research did not receive any specific grant from any funding agencies.

\section{Ethics Approval}

Ethics approval was gained from the Royal Adelaide Hospital Research Ethics Committee to use Figure 2 for demonstrating CBV calculations in DSC-MRI. (R20171002).

\section{References}

[1] Voglein, J., Tuttenberg, J., Weimer, M., et al. (2011) Treatment Monitoring in Gliomas: Comparison of Dynamic Susceptibility-Weighted Contrast-Enhanced and Spectroscopic MRI Techniques for Identifying Treatment Failure. Investigative Radiology, 46, 390-400. https://doi.org/10.1097/RLI.0b013e31820e1511

[2] Ho, V.K., Reijneveld, J.C., Enting, R.H., et al. (2014) Changing Incidence and Improved Survival of Gliomas. European Journal of Cancer, 50, 2309-2318. https://doi.org/10.1016/j.ejca.2014.05.019

[3] Stupp, R., Mason, W.P., van den Bent, M.J., et al. (2005) Radiotherapy plus Concomitant and Adjuvant Temozolomide for Glioblastoma. The New England Journal of Medicine, 352, 987-996. https://doi.org/10.1056/NEJMoa043330

[4] Brandes, A.A., Franceschi, E., Tosoni, A., et al. (2008) Mgmt Promoter Methylation Status Can Predict the Incidence and Outcome of Pseudo Progression after Concomitant Radiochemotherapy in Newly Diagnosed Glioblastoma Patients. Journal of Clinical Oncology, 26, 2192-2197. https://doi.org/10.1200/JCO.2007.14.8163

[5] Brandsma, D., Stalpers, L., Taal, W., Sminia, P. and van den Bent, M.J. (2008) Clinical Features, Mechanisms, and Management of Pseudo Progression in Malignant Gliomas. The Lancet Oncology, 9, 453-461. https://doi.org/10.1016/S1470-2045(08)70125-6

[6] Gerstner, E.R., McNamara, M.B., Norden, A.D., Lafrankie, D. and Wen, P.Y. (2009) Effect of Adding Temozolomide to Radiation Therapy on the Incidence of Pseudo-Progression. Journal of Neuro-Oncology, 94, 97-101.

https://doi.org/10.1007/s11060-009-9809-4

[7] Perry, J.R., Belanger, K., Mason, W.P., et al. (2010) Phase II Trial of Continuous Dose-Intense Temozolomide in Recurrent Malignant Glioma: Rescue Study. Journal of Clinical Oncology, 28, 2051-2057. https://doi.org/10.1200/JCO.2009.26.5520

[8] Reardon, D.A., Galanis, E., DeGroot, J.F., et al. (2011) Clinical Trial End Points for High-Grade Glioma: The Evolving Landscape. Neuro-Oncology, 13, 353-361. https://doi.org/10.1093/neuonc/noq203

[9] van den Bent, M.J., Vogelbaum, M.A., Wen, P.Y., Macdonald, D.R. and Chang, S.M. (2009) End Point Assessment in Gliomas: Novel Treatments Limit Usefulness of Classical Macdonald's Criteria. Journal of Clinical Oncology, 27, 2905-2908. https://doi.org/10.1200/JCO.2009.22.4998

[10] Macdonald, D.R., Cascino, T.L., Schold Jr., S.C. and Cairncross, J.G. (1990) Response Criteria for Phase II Studies of Supratentorial Malignant Glioma. Journal of 
Clinical Oncology, 8, 1277-1280. https://doi.org/10.1200/JCO.1990.8.7.1277

[11] Wen, P.Y., Macdonald, D.R., Reardon, D.A., et al. (2010) Updated Response Assessment Criteria for High-Grade Gliomas: Response Assessment in Neuro-Oncology Working Group. Journal of Clinical Oncology, 28, 1963-1972. https://doi.org/10.1200/JCO.2009.26.3541

[12] Nasseri, M., Gahramanov, S., Netto, J.P., et al. (2014) Evaluation of Pseudo Progression in Patients with Glioblastoma Multiforme Using Dynamic Magnetic Resonance Imaging with Ferumoxytol Calls RANO Criteria into Question. Neuro-Oncology, 16, 1146-1154. https://doi.org/10.1093/neuonc/not328

[13] Prat, R., Galeano, I., Lucas, A., et al. (2010) Relative Value of Magnetic Resonance Spectroscopy, Magnetic Resonance Perfusion, and 2- $\left({ }^{18} \mathrm{~F}\right)$ Fluoro-2-Deoxy-D-Glucose Positron Emission Tomography for Detection of Recurrence or Grade Increase in Gliomas. Journal of Clinical Neuroscience, 17, 50-53. https://doi.org/10.1016/j.jocn.2009.02.035

[14] Alexiou, G.A., Zikou, A., Tsiouris, S., et al. (2014) Comparison of Diffusion Tensor, Dynamic Susceptibility Contrast MRI and ${ }^{99 \mathrm{~m}}$ Tc-Tetrofosmin Brain SPECT for the Detection of Recurrent High-Grade Glioma. Magnetic Resonance Imaging, 32, 854-859. https://doi.org/10.1016/j.mri.2014.04.013

[15] Baek, H.J., Kim, H.S., Kim, N., Choi, Y.J. and Kim, Y.J. (2012) Percent Change of Perfusion Skewness and Kurtosis: A Potential Imaging Biomarker for Early Treatment Response in Patients with Newly Diagnosed Glioblastomas. Radiology, 264, 834-843. https://doi.org/10.1148/radiol.12112120

[16] Barajas Jr., R.F., Chang, J.S., Segal, M.R., et al. (2009) Differentiation of Recurrent Glioblastoma Multiforme from Radiation Necrosis after External Beam Radiation Therapy with Dynamic Susceptibility-Weighted Contrast-Enhanced Perfusion MR Imaging. Radiology, 253, 486-496. https://doi.org/10.1148/radiol.2532090007

[17] Blasel, S., Zagorcic, A., Jurcoane, A., et al. (2016) Perfusion MRI in the Evaluation of Suspected Glioblastoma Recurrence. Journal of Neuroimaging, 26, 116-123. https://doi.org/10.1111/jon.12247

[18] Boxerman, J.L., Ellingson, B.M., Jeyapalan, S., et al. (2017) Longitudinal DSC-MRI for Distinguishing Tumor Recurrence from Pseudo Progression in Patients with a High-Grade Glioma. American Journal of Clinical Oncology, 40, 228-234. https://doi.org/10.1097/COC.0000000000000156

[19] Choi, Y.J., Kim, H.S., Jahng, G.H., Kim, S.J. and Suh, D.C. (2013) Pseudo Progression in Patients with Glioblastoma: Added Value of Arterial Spin Labeling to Dynamic Susceptibility Contrast Perfusion MR Imaging. Acta Radiologica, 54, 448-454. https://doi.org/10.1177/0284185112474916

[20] Dandois, V., Rommel, D., Renard, L., Jamart, J. and Cosnard, G. (2010) Substitution of 11C-Methionine PET by Perfusion MRI during the Follow-Up of Treated High-Grade Gliomas: Preliminary Results in Clinical Practice. Journal of Neuroradiology, 37, 89-97. https://doi.org/10.1016/j.neurad.2009.04.005

[21] Di Costanzo, A., Scarabino, T., Trojsi, F., et al. (2014) Recurrent Glioblastoma Multiforme versus Radiation Injury: A Multiparametric ${ }_{3}$-T MR Approach. La Radiologia Medica, 119, 616-624. https://doi.org/10.1007/s11547-013-0371-y

[22] D’Souza, M.M., Sharma, R., Jaimini, A., et al. (2014) 11C-MET PET/CT and Advanced MRI in the Evaluation of Tumor Recurrence in High-Grade Gliomas. Clinical Nuclear Medicine, 39, 791-798. https://doi.org/10.1097/RLU.0000000000000532

[23] Hu, L.S., Baxter, L.C., Smith, K.A., et al. (2009) Relative Cerebral Blood Volume Values to Differentiate High-Grade Glioma Recurrence from Posttreatment Radia- 
tion Effect: Direct Correlation between Image-Guided Tissue Histopathology and Localized Dynamic Susceptibility-Weighted Contrast-Enhanced Perfusion MR Imaging Measurements. American Journal of Neuroradiology, 30, 552-558. https://doi.org/10.3174/ajnr.A1377

[24] Hu, L.S., Eschbacher, J.M., Heiserman, J.E., et al. (2012) Reevaluating the Imaging Definition of Tumor Progression: Perfusion MRI Quantifies Recurrent Glioblastoma Tumor Fraction, Pseudo Progression, and Radiation Necrosis to Predict Survival. Neuro-Oncology, 14, 919-930. https://doi.org/10.1093/neuonc/nos112

[25] Hu, X., Wong, K.K., Young, G.S., Guo, L. and Wong, S.T. (2011) Support Vector Machine Multiparametric MRI Identification of Pseudo Progression from Tumor Recurrence in Patients with Resected Glioblastoma. Journal of Magnetic Resonance Imaging, 33, 296-305. https://doi.org/10.1002/jmri.22432

[26] Kim, H.S., Kim, J.H., Kim, S.H., Cho, K.G. and Kim, S.Y. (2010) Posttreatment High-Grade Glioma: Usefulness of Peak Height Position with Semiquantitative MR Perfusion Histogram Analysis in an Entire Contrast-Enhanced Lesion for Predicting Volume Fraction of Recurrence. Radiology, 256, 906-915.

https://doi.org/10.1148/radiol.10091461

[27] Kim, T.H., Yun, T.J., Park, C.K., et al. (2017) Combined Use of Susceptibility Weighted Magnetic Resonance Imaging Sequences and Dynamic Susceptibility Contrast Perfusion Weighted Imaging to Improve the Accuracy of the Differential Diagnosis of Recurrence and Radionecrosis in High-Grade Glioma Patients. Oncotarget, 8, 20340-20353. https://doi.org/10.18632/oncotarget.13050

[28] Kim, Y.H., Oh, S.W., Lim, Y.J., et al. (2010) Differentiating Radiation Necrosis from Tumor Recurrence in High-Grade Gliomas: Assessing the Efficacy of ${ }^{18}$ F-FDG PET, ${ }^{11}$ C-Methionine PET and Perfusion MRI. Clinical Neurology and Neurosurgery, 112, 758-765. https://doi.org/10.1016/j.clineuro.2010.06.005

[29] Kong, D.S., Kim, S.T., Kim, E.H., et al. (2011) Diagnostic Dilemma of Pseudo Progression in the Treatment of Newly Diagnosed Glioblastomas: The Role of Assessing Relative Cerebral Blood Flow Volume and Oxygen-6-Methylguanine-DNA Methyltransferase Promoter Methylation Status. American Journal of Neuroradiology, 32, 382-387. https://doi.org/10.3174/ajnr.A2286

[30] Mangla, R., Singh, G., Ziegelitz, D., et al. (2010) Changes in Relative Cerebral Blood Volume 1 Month after Radiation-Temozolomide Therapy Can Help Predict Overall Survival in Patients with Glioblastoma. Radiology, 256, 575-584.

https://doi.org/10.1148/radiol.10091440

[31] Ozsunar, Y., Mullins, M.E., Kwong, K., et al. (2010) Glioma Recurrence versus Radiation Necrosis? A Pilot Comparison of Arterial Spin-Labeled, Dynamic Susceptibility Contrast Enhanced MRI, and FDG-PET Imaging. Academic Radiology, 17, 282-290. https://doi.org/10.1016/j.acra.2009.10.024

[32] Prager, A.J., Martinez, N., Beal, K., Omuro, A., Zhang, Z. and Young, R.J. (2015) Diffusion and Perfusion MRI to Differentiate Treatment-Related Changes Including Pseudo Progression from Recurrent Tumors in High-Grade Gliomas with Histopathologic Evidence. American Journal of Neuroradiology, 36, 877-885. https://doi.org/10.3174/ajnr.A4218

[33] Sacconi, B., Raad, R.A., Lee, J., et al. (2016) Concurrent Functional and Metabolic Assessment of Brain Tumors Using Hybrid PET/MR Imaging. Journal of Neuro-Oncology, 127, 287-293. https://doi.org/10.1007/s11060-015-2032-6

[34] Seeger, A., Braun, C., Skardelly, M., et al. (2013) Comparison of Three Different MR Perfusion Techniques and MR Spectroscopy for Multiparametric Assessment in 
Distinguishing Recurrent High-Grade Gliomas from Stable Disease. Academic Radiology, 20, 1557-1565. https://doi.org/10.1016/j.acra.2013.09.003

[35] Shin, K.E., Ahn, K.J., Choi, H.S., et al. (2014) DCE and DSC MR Perfusion Imaging in the Differentiation of Recurrent Tumour from Treatment-Related Changes in Patients with Glioma. Clinical Radiology, 69, e264-e272. https://doi.org/10.1016/j.crad.2014.01.016

[36] Snelling, B., Shah, A.H., Buttrick, S. and Benveniste, R. (2017) The Use of MR Perfusion Imaging in the Evaluation of Tumor Progression in Gliomas. Journal of Korean Neurosurgical Society, 60, 15-20. https://doi.org/10.3340/jkns.2016.0102.001

[37] Song, Y.S., Choi, S.H., Park, C.K., et al. (2013) True Progression versus Pseudo Progression in the Treatment of Glioblastomas: A Comparison Study of Normalized Cerebral Blood Volume and Apparent Diffusion Coefficient by Histogram Analysis. Korean Journal of Radiology, 14, 662-672. https://doi.org/10.3348/kjr.2013.14.4.662

[38] Wang, S., Martinez-Lage, M., Sakai, Y., et al. (2016) Differentiating Tumor Progression from Pseudo progression in Patients with Glioblastomas Using Diffusion Tensor Imaging and Dynamic Susceptibility Contrast MRI. American Journal of Neuroradiology, 37, 28-36. https://doi.org/10.3174/ajnr.A4474

[39] Young, R.J., Gupta, A., Shah, A.D., et al. (2013) MRI Perfusion in Determining Pseudo Progression in Patients with Glioblastoma. Clinical Imaging, 37, 41-49. https://doi.org/10.1016/j.clinimag.2012.02.016

[40] Chung, W.J., Kim, H.S., Kim, N., Choi, C.G. and Kim, S.J. (2013) Recurrent Glioblastoma: Optimum Area under the Curve Method Derived from Dynamic Contrast-Enhanced T1-Weighted Perfusion MR Imaging. Radiology, 269, 561-568. https://doi.org/10.1148/radiol.13130016

[41] Hamilton, J.D., Lin, J., Ison, C., et al. (2015) Dynamic Contrast-Enhanced Perfusion Processing for Neuroradiologists: Model-Dependent Analysis May Not Be Necessary for Determining Recurrent High-Grade Glioma versus Treatment Effect. AJNR American Journal of Neuroradiology, 36, 686-693. https://doi.org/10.3174/ajnr.A4190

[42] Heo, Y.J., Kim, H.S., Park, J.E., Choi, C.G. and Kim, S.J. (2015) Uninterpretable Dynamic Susceptibility Contrast-Enhanced Perfusion MR Images in Patients with Post-Treatment Glioblastomas: Cross-Validation of Alternative Imaging Options. PLOS ONE, 10, e0136380. https://doi.org/10.1371/journal.pone.0136380

[43] Kim, H.S., Goh, M.J., Kim, N., Choi, C.G., Kim, S.J. and Kim, J.H. (2014) Which Combination of MR Imaging Modalities Is Best for Predicting Recurrent Glioblastoma? Study of Diagnostic Accuracy and Reproducibility. Radiology, 273, 831-843. https://doi.org/10.1148/radiol.14132868

[44] Larsen, V.A., Simonsen, H.J., Law, I., Larsson, H.B. and Hansen, A.E. (2013) Evaluation of Dynamic Contrast-Enhanced T1-Weighted Perfusion MRI in the Differentiation of Tumor Recurrence from Radiation Necrosis. Neuroradiology, 55, 361-369. https://doi.org/10.1007/s00234-012-1127-4

[45] Narang, J., Jain, R., Arbab, A.S., et al. (2011) Differentiating Treatment-Induced Necrosis from Recurrent/Progressive Brain Tumor Using Nonmodel-Based Semiquantitative Indices Derived from Dynamic Contrast-Enhanced T1-Weighted MR Perfusion. Neuro-Oncology, 13, 1037-1046. https://doi.org/10.1093/neuonc/nor075

[46] Suh, C.H., Kim, H.S., Choi, Y.J., Kim, N. and Kim, S.J. (2013) Prediction of Pseudo progression in Patients with Glioblastomas Using the Initial and Final Area under the Curves Ratio Derived from Dynamic Contrast-Enhanced T1-Weighted Perfusion MR Imaging. American Journal of Neuroradiology, 34, 2278-2286. 
https://doi.org/10.3174/ajnr.A3634

[47] Thomas, A.A., Arevalo-Perez, J., Kaley, T., et al. (2015) Dynamic Contrast Enhanced T1 MRI Perfusion Differentiates Pseudo Progression from Recurrent Glioblastoma. Journal of Neuro-Oncology, 125, 183-190. https://doi.org/10.1007/s11060-015-1893-Z

[48] Yoo, R.E., Choi, S.H., Kim, T.M., et al. (2017) Dynamic Contrast-Enhanced MR Imaging in Predicting Progression of Enhancing Lesions Persisting after Standard Treatment in Glioblastoma Patients: A Prospective Study. European Radiology, 27, 3156-3166. https://doi.org/10.1007/s00330-016-4692-9

[49] Yoon, R.G., Kim, H.S., Paik, W., Shim, W.H., Kim, S.J. and Kim, J.H. (2017) Different Diagnostic Values of Imaging Parameters to Predict Pseudo Progression in Glioblastoma Subgroups Stratified by MGMT Promoter Methylation. European Radiology, 27, 255-266. https://doi.org/10.1007/s00330-016-4346-y

[50] Park, K.J., Kim, H.S., Park, J.E., Shim, W.H., Kim, S.J. and Smith, S.A. (2016) Added Value of Amide Proton Transfer Imaging to Conventional and Perfusion MR Imaging for Evaluating the Treatment Response of Newly Diagnosed Glioblastoma. European Radiology, 26, 4390-4403. https://doi.org/10.1007/s00330-016-4261-2

[51] Hu, L.S., Kelm, Z., Korfiatis, P., et al. (2015) Impact of Software Modeling on the Accuracy of Perfusion MRI in Glioma. American Journal of Neuroradiology, 36, 2242-2249. https://doi.org/10.3174/ajnr.A4451

[52] Kelm, Z.S., Korfiatis, P.D., Lingineni, R.K., et al. (2015) Variability and Accuracy of Different Software Packages for Dynamic Susceptibility Contrast Magnetic Resonance Imaging for Distinguishing Glioblastoma Progression from Pseudo Progression. Journal of Medical Imaging, 2, Article ID: 026001. https://doi.org/10.1117/1.JMI.2.2.026001

[53] Shiroishi, M.S., Boxerman, J.L. and Pope, W.B. (2016) Physiologic MRI for Assessment of Response to Therapy and Prognosis in Glioblastoma. Neuro-Oncology, 18, 467-478. https://doi.org/10.1093/neuonc/nov179

[54] Tofts, P.S., Brix, G., Buckley, D.L., et al. (1999) Estimating Kinetic Parameters from Dynamic Contrast-Enhanced $\mathrm{T}_{1}$-Weighted MRI of a Diffusable Tracer: Standardized Quantities and Symbols. Journal of Magnetic Resonance Imaging, 10, 223-232. https://doi.org/10.1002/(SICI)1522-2586(199909)10:3<223::AID-JMRI2>3.0.CO;2-S

[55] Patlak, C.S. and Blasberg, R.G. (1985) Graphical Evaluation of Blood-to-Brain Transfer Constants from Multiple-Time Uptake Data. Generalizations. Journal of Cerebral Blood Flow \& Metabolism, 5, 584-590. https://doi.org/10.1038/jcbfm.1985.87

[56] Bonicelli, C., Bacci, A., Agati, R. and Leonardi, M. (2009) Potential of High Field Functional MRI in the Neuroradiological Diagnosis of Brain Tumours. The Neuroradiology Journal, 22, 534-545. https://doi.org/10.1177/197140090902200504

[57] Scarabino, T., Giannatempo, G.M., Popolizio, T., et al. (2007) 3.0-T Functional Brain Imaging: A 5-Year Experience. La Radiologia Medica, 112, 97-112. https://doi.org/10.1007/s11547-007-0124-x

[58] Hu, L.S., Baxter, L.C., Pinnaduwage, D.S., et al. (2010) Optimized Preload Leakage-Correction Methods to Improve the Diagnostic Accuracy of Dynamic Susceptibility-Weighted Contrast-Enhanced Perfusion MR Imaging in Posttreatment Gliomas. American Journal of Neuroradiology, 31, 40-48. https://doi.org/10.3174/ajnr.A1787

[59] Paulson, E.S. and Schmainda, K.M. (2008) Comparison of Dynamic Susceptibili- 
ty-Weighted Contrast-Enhanced MR Methods: Recommendations for Measuring Relative Cerebral Blood Volume in Brain Tumors. Radiology, 249, 601-613. https://doi.org/10.1148/radiol.2492071659

[60] Gahramanov, S., Muldoon, L.L., Varallyay, C.G., et al. (2013) Pseudo Progression of Glioblastoma after Chemo- and Radiation Therapy: Diagnosis by Using Dynamic Susceptibility-Weighted Contrast-Enhanced Perfusion MR Imaging with Ferumoxytol versus Gadoteridol and Correlation with Survival. Radiology, 266, 842-852. https://doi.org/10.1148/radiol.12111472

[61] Roberts, C., Issa, B., Stone, A., et al. (2006) Comparative Study into the Robustness of Compartmental Modeling and Model-Free Analysis in DCE-MRI Studies. Journal of Magnetic Resonance Imaging, 23, 554-563.

https://doi.org/10.1002/jmri.20529

[62] Nyberg, E., Honce, J., Kleinschmidt-DeMasters, B.K., Shukri, B., Kreidler, S. and Nagae, L. (2016) Arterial Spin Labeling: Pathologically Proven Superiority over Conventional MRI for Detection of High-Grade Glioma Progression after Treatment. The Neuroradiology Journal, 29, 377-383.

https://doi.org/10.1177/1971400916665375

[63] Chahal, M., Xu, Y., Lesniak, D., et al. (2010) MGMT Modulates Glioblastoma Angiogenesis and Response to the Tyrosine Kinase Inhibitor Sunitinib. Neuro-Oncology, 12, 822-833. https://doi.org/10.1093/neuonc/noq017

[64] Bernstein, M. and Parrent, A.G. (1994) Complications of CT-Guided Stereotactic Biopsy of Intra-Axial Brain Lesions. Journal of Neurosurgery, 81, 165-168. https://doi.org/10.3171/jns.1994.81.2.0165

[65] Malone, H., Yang, J., Hershman, D.L., Wright, J.D., Bruce, J.N. and Neugu, A.I. (2015) Complications Following Stereotactic Needle Biopsy of Intracranial Tumors. World Neurosurgery, 84, 1084-1089. https://doi.org/10.1016/j.wneu.2015.05.025

[66] McGirt, M.J., Woodworth, G.F., Coon, A.L., et al. (2005) Independent Predictors of Morbidity after Image-Guided Stereotactic Brain Biopsy: A Risk Assessment of 270 Cases. Journal of Neurosurgery, 102, 897-901. https://doi.org/10.3171/jns.2005.102.5.0897

[67] Forsyth, P.A., Kelly, P.J., Cascino, T.L., et al. (1995) Radiation Necrosis or Glioma Recurrence: Is Computer-Assisted Stereotactic Biopsy Useful? Journal of Neurosurgery, 82, 436-444. https://doi.org/10.3171/jns.1995.82.3.0436 